\title{
The dark side of immunotherapy
}

\author{
Nwanneka Okwundu ${ }^{1}$, Douglas Grossman ${ }^{1,2}$, Siwen Hu-Lieskovan ${ }^{1,3}$, Kenneth F. Grossmann ${ }^{1,3}$, \\ Umang Swami ${ }^{1,3} \wedge$
}

${ }^{1}$ Huntsman Cancer Institute, University of Utah Health Sciences Center, Salt Lake City, UT, USA; ${ }^{2}$ Department of Dermatology, University of Utah Health Sciences Center, Salt Lake City, UT, USA; ${ }^{3}$ Department of Medicine, University of Utah Health Sciences Center, Salt Lake City, UT, USA Contributions: (I) Conception and design: U Swami; (II) Administrative support: All authors; (III) Provision of study materials or patients: All authors; (IV) Collection and assembly of data: N Okwundu, U Swami; (V) Data analysis and interpretation: All authors; (VI) Manuscript writing: All authors; (VII) Final approval of manuscript: All authors.

Correspondence to: Umang Swami, MD, MS. Assistant Professor, Huntsman Cancer Institute, University of Utah (NCI-CCC), 2000 Circle of Hope Drive Research North Bldg Suite No. 2121, Salt Lake City, UT 84112, USA. Email: umang.swami@hci.utah.edu.

\begin{abstract}
Immunotherapy has broadened the therapeutic scope and response for many cancer patients with drugs that are generally of higher efficacy and less toxicity than prior therapies. Multiple classes of immunotherapies such as targeted antibodies and immune checkpoint inhibitors (ICI), cell-based immunotherapies, immunomodulators, vaccines, and oncolytic viruses have been developed to help the immune system target and destroy malignant tumors. ICI targeting programmed cell death protein-1 (PD-1) or its ligand (PD-L1) are among the most effective immunotherapy agents and are a major focus of current investigations. They have received approval for at least 16 different tumor types as well as for unresectable or metastatic tumors with microsatellite instability-high (MSI-H) or mismatch repair deficiency or with high tumor mutational burden (defined as $\geq 10$ mutations/megabase). However, it is important to recognize that immunotherapy may be associated with significant adverse events. To summarize these events, we conducted a PubMed and Google Scholar database search through April 2020 for manuscripts evaluating treatmentrelated adverse events and knowledge gaps associated with the use of immunotherapy. Reviewed topics include immune-related adverse events (irAEs), toxicities on combining immunotherapy with other agents, disease reactivation such as tuberculosis (TB) and sarcoid-like granulomatosis, tumor hyperprogression (HPD), financial toxicity, challenges in special patient populations such as solid organ transplant recipients and those with auto-immune diseases. We also reviewed reports of worse or even lethal outcomes compared to other oncologic therapies in certain scenarios and summarized biomarkers predicting adverse events.
\end{abstract}

Keywords: Immunotherapy; immune-related adverse events (irAEs); autoimmune; hyperprogression (HPD); solid organ transplant

Submitted Jun 16, 2020. Accepted for publication Oct 28, 2020.

doi: $10.21037 /$ atm-20-4750

View this article at: http://dx.doi.org/10.21037/atm-20-4750

\section{Introduction}

Immunotherapy represents a breakthrough in oncology that has radically changed the therapeutic management of numerous cancer types. Multiple classes of agents have been developed to enhance the ability of the immune system to target and destroy malignant tumors but may also be associated with immune-related adverse events (irAEs) (1). These include immune checkpoint inhibitors (ICI), cell transfer therapies, vaccines, and immune system modulators. These agents, their mechanisms of action, and common irAEs are summarized in Table 1.

\footnotetext{
$\wedge$ ORCID: 0000-0003-3518-0411.
} 
Table 1 Selected approved cancer immunotherapies, mechanisms of action, and adverse effects

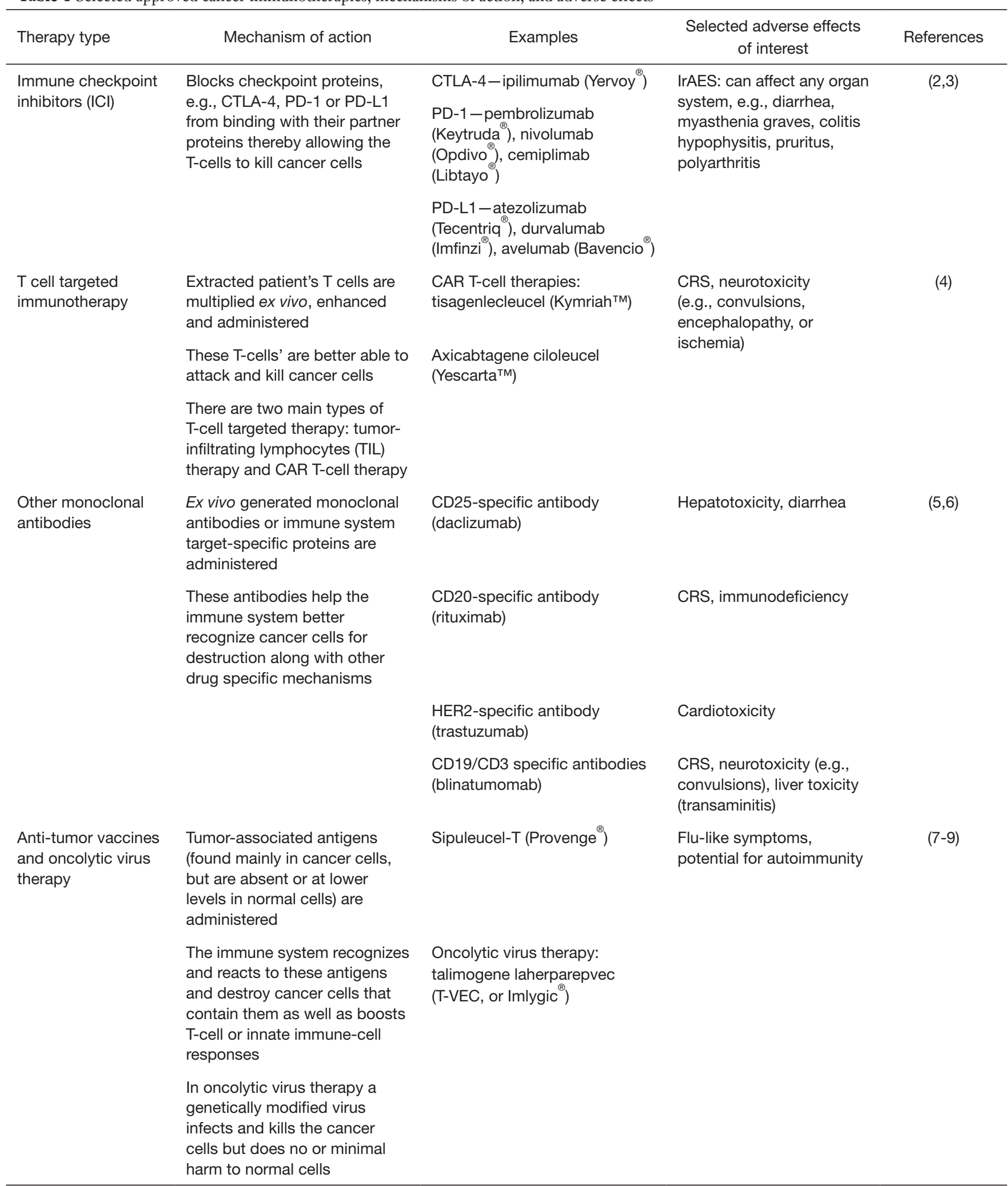

Table 1 (continued) 
Table 1 (continued)

\begin{tabular}{|c|c|c|c|c|}
\hline Therapy type & Mechanism of action & Examples & $\begin{array}{c}\text { Selected adverse effects } \\
\text { of interest }\end{array}$ & References \\
\hline \multirow[t]{4}{*}{ Immunomodulators } & $\begin{array}{l}\text { Immune-modulating agents } \\
\text { such as cytokines and BCG are } \\
\text { administered }\end{array}$ & Thalidomide $\left(\right.$ Thalomid $^{\otimes}$ ) & $\begin{array}{l}\text { Teratogenic, } \\
\text { myelosuppression }\end{array}$ & $(9-11)$ \\
\hline & $\begin{array}{l}\text { They enhance the body's } \\
\text { immune response against } \\
\text { cancer or reduce side effect of } \\
\text { chemotherapy }\end{array}$ & Lenalidomide (Revlimid ${ }^{\circledR}$ ) & $\begin{array}{l}\text { Neutropenia, diarrhea, } \\
\text { anemia, TLS }\end{array}$ & \\
\hline & & Pomalidomide (Pomalyst ${ }^{\circledR}$ ) & $\begin{array}{l}\text { Thromboembolism, } \\
\text { neurotoxicity, TLS }\end{array}$ & \\
\hline & & Imiquimod (Aldara ${ }^{\circledR}$, Zyclara $\left.^{\circledR}\right)$ & $\begin{array}{l}\text { Dermatitis, cold sores, } \\
\text { headache, flu-like } \\
\text { symptoms }\end{array}$ & \\
\hline $\begin{array}{l}\text { Cellular } \\
\text { immunotherapy }\end{array}$ & $\begin{array}{l}\text { Autologous or allogeneic stem } \\
\text { cells are infused } \\
\text { Treat hematopoietic } \\
\text { malignancies or aid } \\
\text { recovery in cancer patients } \\
\text { immunoablated with very } \\
\text { high doses of radiation therapy, } \\
\text { chemotherapy, } \\
\text { or both }\end{array}$ & $\begin{array}{l}\text { Peripheral blood stem cells } \\
\text { (PBSCs) }\end{array}$ & $\begin{array}{l}\text { Autoimmunity due to off- } \\
\text { target responses, including } \\
\text { uveitis (in melanoma) and } \\
\text { GVHD (in haematopoietic } \\
\text { malignancies) }\end{array}$ & $(9,12)$ \\
\hline
\end{tabular}

BCG, Mycobacterium bovis bacillus Calmette-Guérin; CTLA4, cytotoxic T-lymphocyte antigen 4; PD-1, programmed cell death protein 1; PD-L1, programmed death ligand 1; CAR T-cell, chimeric antigen receptor T-cell; HER2, human epidermal growth-factor receptor 2; irAEs, immune-related adverse events; TLS, tumor lysis syndrome; CRS, cytokine release syndrome; GVHD, graft versus host disease.

Immunotherapies have improved overall survival (OS) in a broad range of early-stage and advanced cancer types, and these treatments have gained wide acceptance and considerable excitement in clinical practice. Antibodies targeting programmed cell death protein-1 (PD-1) or its ligand (PD-L1) are the most effective of the ICIs. As per a recent report, at least nine PD-1/PD-L1-directed agents have reached the clinics globally for the treatment of 16 different cancer types, and microsatellite instabilityhigh (MSI-H) or mismatch repair deficient (dMMR) solid tumors (13).

However, these novel agents are not without their unique downsides which include high cost, immune toxicities, hyperprogression (HPD), reactivation of certain diseases, limitations in certain populations, and unexpected worse outcomes in certain malignancies. This review summarizes these negative health impacts and problems associated with the use of immunotherapy (Figure 1).

\section{irAEs}

Although immunotherapy has proven to be more effective and less toxic compared to chemotherapy in multiple cancer types, unexpected adverse events have been observed that are likely related to their mechanism of action (14). These therapies generally work by relieving inhibition of activated T-lymphocytes in lymph nodes and peripheral tissues, resulting in enhanced lymphocyte activation and propagation of T-cell mediated destruction of normal cells expressing self-antigens and induce inflammatory and autoimmune responses leading to irAEs (6). Clinically, irAEs may manifest as distinct symptoms with respect to organ 


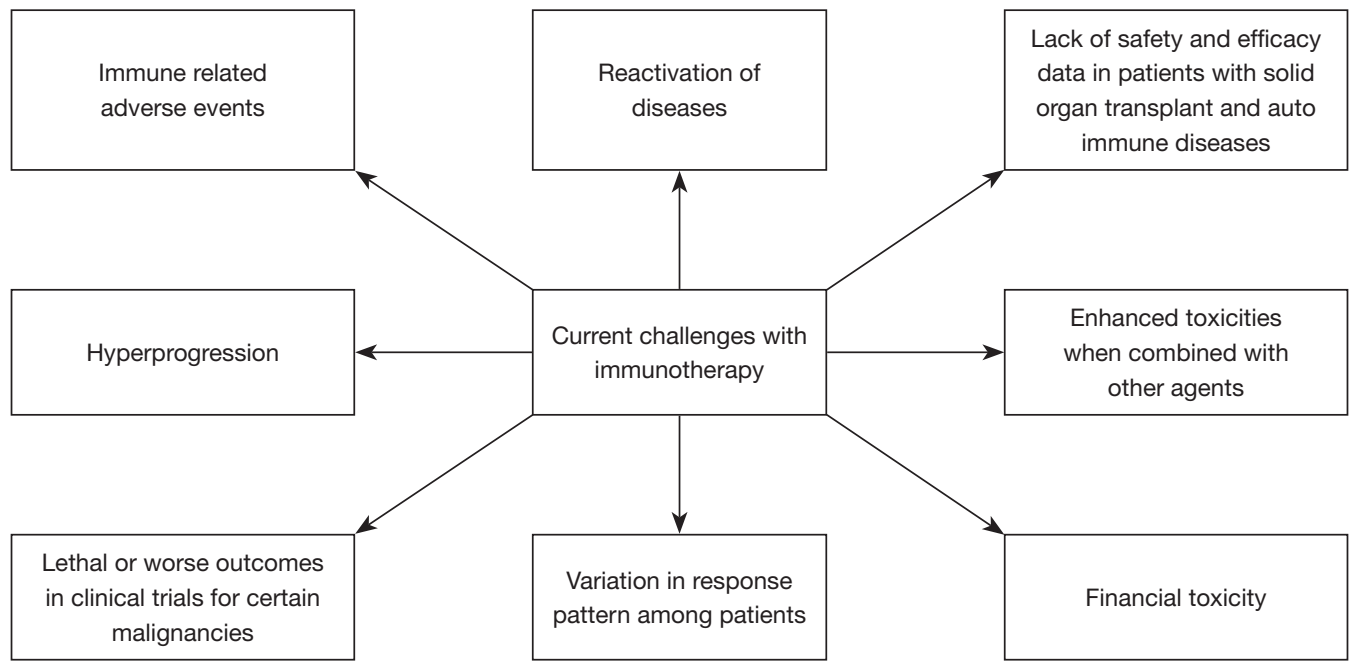

Figure 1 Summary of current challenges with cancer immunotherapy.

involvement, pattern of onset, and level of severity which may lead to severe and even life-threatening complications in patients that may necessitate early termination of an otherwise beneficial treatment. Up to $95 \%$ of patients receiving immunotherapy may experience irAEs (15-19) mainly due to immune dysregulation targeting normal tissue antigens (20-22). In initial reports, the organs most frequently involved were the skin (pruritus and cutaneous rash), joints, thyroid, and gastrointestinal tract (colitis and diarrhea). Subsequently, irAEs were identified in the liver (autoimmune hepatitis), non-thyroid endocrine glands (hypophysitis and adrenal insufficiency), lungs and nervous system (myasthenia gravis and encephalitis). Finally, myositis/myocarditis, nephritis, and even hematological irAEs were reported (23-25). Interestingly, irAEs may be idiosyncratic as different drugs and doses used for different cancers may result in different adverse events of unpredictable severity $(23,26,27)$.

The irAEs induced by ICI therapy are associated with a fatality rate of around $5 \%$. In an analysis of co-morbidities in 21 patients on ICI with irAEs related death, 12 had hypertension and 6 had other cardiac conditions; 2 patients had a preexisting autoimmune disease (AD) (Graves' disease). In addition, patients with fatal toxic effects had similar sex distribution but were older than those without fatal toxic effects (median, 70 vs. 62 years; absolute difference, $8 ; \mathrm{P}=0.009$ ) (28). The most common cause of death from ICI-related irAEs is (ICI)-related pneumonitis (ICI-P). The incidence of ICI-P in phase III trials was between $<0.5 \%$ and $10 \%$ for all grades and has been found to be higher in lung cancer (1-6\%) than in other cancers (0.1-4\%) (29-37). In a meta-analysis including all cancer types and ICI, ICI-P of all grades was observed in $2.6 \%$ of patients and emerged as the fourth leading cause of ICIinduced irAEs after skin eruptions (13.9\%), hepatitis (6.5\%), thyroid disorders (5.1\%), and colitis (2.3\%) (38). In another meta-analysis, the most common irAEs due to PD- 1 and PD-L1 inhibitors were fatigue (18.3\%), pruritus (10.6\%), and diarrhea $(9.5 \%)$. Though the incidence of all grade ICI-P was $2.79 \%$ it was responsible for $28 \%$ of treatmentrelated deaths (39).

Immune-mediated myocarditis is the most common type of cardiotoxicity occurring with immunotherapy. Although the incidence is low partly due to under-recognition, the cardiovascular-specific mortality rate is relatively high and varies with different therapies. In patients who developed cardiotoxicity, the cardiovascular-specific mortality was $12 \%$ in patients receiving nivolumab monotherapy, $19 \%$ in pembrolizumab monotherapy cohorts, and $65 \%$ in combined CTLA-4 and PD-1 blockade (ipilimumab and nivolumab combination) (40).

Endocrine adverse events though rare could be lifethreatening and hence are noteworthy irAEs. Blocking inhibitory molecules on activated T cells by ICI not only increases the killing of tumor cells it could also lead to infringement of self-tolerance, enabling $\mathrm{T}$ cells to act against self-antigens (41). The most frequent autoimmune endocrinopathies involve the pituitary and thyroid 
glands (42). Thyroid abnormalities such as hypothyroidism, thyrotoxicosis, painless thyroiditis, or even thyroid storm (43) are present in $1-6 \%$ of patients administered with ICI $(43,44)$. Patients with endocrine irAE can be critically ill at presentation. For instance, primary adrenal insufficiency during immunotherapy is a medical emergency requiring hydrocortisone replacement. Suggestive acute symptoms include hypotension, hyponatremia, nausea, vomiting, diarrhea, asthenia, weight loss, dehydration, fever, abdominal pain, cramp and muscle pain. Current data suggest that treatment needs to be life-long, even after termination of immunotherapy (45).

Furthermore, patients could develop autoimmune type 1 diabetes and also often present emergently with severe hyperglycemia or diabetic ketoacidosis (DKA) with elevated $\mathrm{HbA}_{1 \mathrm{c}}$. Time from drug administration to diabetes onset may span 1 week to 5 months (41). In a systematic review of 90 patient cases treated with anti-PD-1 or anti-PD-L1 as monotherapy $(79 \%)$ or in combination with CTLA-4 blockade (15\%), diabetes mellitus was diagnosed after 4.5 cycles (range, 1-17); while earlier with combination ICI at 2.7 cycles (range, $1-5$ ) on average. Islet autoantibodies were positive in $53 \%(47 / 88)$ of patients with a predominance of glutamic acid decarboxylase antibodies. Susceptible HLA genotypes were present in 65\% (mostly DR4) (46). Hence, the use of anti-GAD serology in aiding the diagnosis is not recommended as only half of the patients were positive although this can potentially be used to confirm the diagnosis if positive (47). Incidence is estimated to be at $0.4-0.6 \%$ in clinical trials $(44,48,49)$, while a recent study reported a prevalence of $0.9 \%$ among 2,960 patients treated by immunotherapy (50). Immune-related endocrine toxicities such as thyroid dysfunctions, hypophysitis, adrenal insufficiency, and type 1 diabetes mellitus are irreversible in approximately $50 \%$ of patients (51).

Most of the irAEs symptoms are generally wellcontrolled by discontinuing the drug and/or adding systemic glucocorticoids or steroid-sparing regimens (e.g., anti-tumor necrosis factor-alpha (TNF- $\alpha$ ) agents or mycophenolate mofetil). Systemic steroids are usually administered for months and sometimes multiple courses are needed due to repeat flares of these irAEs after taper. This makes the patients immunocompromised for a prolonged period, putting them at risk of infections. In addition, corticosteroid-associated adverse events such as myopathy, gastritis, diabetes mellitus, hypertension, Cushing's syndrome and osteoporosis remain a cause of concern (52).

\section{Biomarkers for irAEs}

Certain biomarkers have been found in studies to be independently predictive of irAEs such as female sex and low baseline cytokine levels. Low IL-6 levels were associated with increased OS in a study of 140 patients with melanoma treated with anti-CTLA-4 (53). In patients with locally advanced or metastatic melanoma an association between ipilimumab-induced colitis and reduced baseline levels of circulating IL-6, IL-8, soluble IL-2 receptor (sCD25), and increased IL-17 has been demonstrated $(54,55)$. Intriguingly, there is a likelihood that the net increase in these proinflammatory cytokines from ICI therapy actually determines immune toxicity. On therapy increases in IL-6 (56) or in soluble cluster of differentiation 163 (sCD163) (57) in two studies of melanoma cohorts treated with nivolumab monotherapy were predictive of psoriasiform dermatitis and a variety of irAEs, respectively.

In addition, the existence of autoantibodies may predict the development of endocrine-specific irAEs following checkpoint inhibition. Increased pretreatment levels of serum antithyroglobulin antibodies was significantly associated with subsequent autoimmune thyroid dysfunction (odds ratio, 26.5; 95\% CI, 8.18-85.8) in a multivariate analysis of patients with advanced solid tumors treated with nivolumab (58). Another study found that the existence of one or more diabetes autoantibodies (against glutamic acid decarboxylase 65 , insulin, islet cells, zinc transporter 8 , or islet antigen 2) prior to ICI therapy precipitated the development of clinical diabetes in a cohort of patients diagnosed with a variety of solid tumors (50). Moreover, proteome array identified baseline serum antibody reactivity in a study of 78 patients treated with ICIs. Machine learning has also identified baseline antibody signatures associated with irAEs with greater than $90 \%$ sensitivity and specificity (59).

Furthermore, the constitution of the gut microbiota may be associated with ICI-induced colitis. For instance, in a prospective study of 34 patients with melanoma treated with anti-CTLA-4, baseline representation of species from the Bacteroidetes phylum was associated with decreased risk of ICI-induced colitis (60). Consequently, some studies have reported a positive association between the on-therapy incidence of irAEs and OS or response for patients treated with anti-CTLA4 (61) or anti-programmed cell death 1 (PD1) (62-64), while others found no association with OS $(65,66)$. 


\section{Reactivation of diseases}

Immunotherapeutic agents may also unmask chronic underlying diseases or opportunistic infections. These include latent tuberculosis (TB), sarcoid granulomatosis, varicella-zoster virus infection, cytomegalovirus-associated enterocolitis, and their potentially serious complications which compromise treatment outcome and patient survival $(67,68)$. In an analysis of 14 cancer patients with active TB infection following PD-1/PD-L1 blockade, only 2 of 14 (14\%) patients who developed active TB infection had received steroids or infliximab for irAEs. Thus, cancer and/ or the immunotherapeutic drugs could be considered the probable etiology of their susceptibility to the TB infection warranting caution (69-77). Hence, in addition to highdose corticosteroids, which when used to manage irAEs can unmask chronic underlying diseases or opportunistic infections, immunotherapeutic drugs could directly reactivate diseases as well.

This direct complication of immunotherapy correlates with the findings in a study which investigated the expression patterns of PD-1 and PD-L- 1 within TBinfected human lung tissue using a human $3 \mathrm{D}$ cell culture model of TB. It was found that PD-1 regulates the immune response in TB (78), and that inhibition of PD-1 accelerates TB bacteria growth through excessive TNF- $\alpha$ secretion (79). Neutralizing TNF- $\alpha$ reversed the augmented growth caused by PD-1-inhbitor therapy (79). This is also the basis for treatment of irAEs associated with ICIs with anti-TNF- $\alpha$ inhibitors (1), suggesting TNF- $\alpha$ may be responsible for both autoimmunity and TB pathology after PD-1 therapy.

Some cancer types and immunotherapeutic drugs have been associated with certain disease reactivation susceptibility. For instance, reactivation of sarcoidlike granulomatosis is associated with ICI treatment for melanoma. Of 19 isolated cases of immunotherapyrelated sarcoid-like granulomatosis (ICI-SLG) reported after initiation of cancer treatment, (80-95), $13(73 \%)$ were melanoma patients. Similarly, ICISLG occurred in 7 out of 509 (1.4\%) stage 3 melanoma patients treated with pembrolizumab, while no ICISLG occurred in the placebo group (96). It was also found that melanoma was the most common cancer type associated with sarcoidosis in a meta-analysis investigating the association between sarcoidosis and cancer, and cytotoxic T-lymphocyte antigen 4 (CTLA-4) inhibitors also had a higher association with ICI-SLG than
PD-1 inhibitors (97). Sarcoid presents a particular problem as it is often mistaken for cancer on scans as a sarcoid flare can be hard to distinguish from progressive disease.

\section{Patients with transplant and auto immune diseases}

Safety and efficacy data are lacking for the use of immunotherapy in patients who have undergone solid organ transplantation (SOT). As a result, these patients have been excluded from clinical trials $(20,98,99)$. There is a concern for the risk of allograft rejection in SOT patients (98). In addition, the immunosuppressive drugs used to manage SOT patients may decrease the immune enhancement and efficacy of immunotherapy (99). In a systematic review of SOT patients treated with ICI, allograft rejection occurred in $37 \%$ and $14 \%$ died as a result of graft rejection. Nivolumab was associated with rejection in about half of patients, while pembrolizumab and ipilimumab were each associated with rejection in about one quarter of patients. Highest rates of graft rejection were seen with kidney (40\%), followed by liver (35\%), and heart (20\%) (100). Graft rejection can be reversed in some patients with the use of high-dose corticosteroids, and occasionally other aggressive immunosuppressive therapies, along with dialysis and ICI discontinuation (101). Furthermore, SOT patients are at increased risk of developing de novo cancers (102-106) which are the second leading cause of death in this patient population (106). The inability to safely use immunotherapy without risk of allograft rejection in these patients poses a significant therapeutic challenge.

In addition, disease exacerbation as well as more severe irAEs may occur in patients with active $\mathrm{AD}$ treated with immunotherapy (98). Immune enhancement by immunotherapy may also exacerbate preexisting $\mathrm{AD}$ in remission. In a systematic review which assessed the use of immunotherapy in the treatment of 123 cancer patients with preexisting $\mathrm{AD}, 75 \%$ reported adverse events and $41 \%$ experienced exacerbation of the preexisting $\mathrm{AD}$ with recurrence manifestation or worsening of prior symptoms (101). Flares were more common in patients with active symptoms $(9 / 15,60 \%)$ as compared to those with subclinical disease $(11 / 37,30 \%)$. Overall, $50 \%$ had disease exacerbation, and $34 \%$ had de novo irAEs. Colitis (14\%) and hypophysitis (5\%) were the most commonly reported de novo irAEs. Therefore, patients with active AD were usually excluded from clinical trials. Now 
multiple clinical trials $(107,108)$ are evaluating ICI in patients with AD.

Potential strategies beyond clinical guidelines have been proposed to lower the risk of irAEs for these specific scenarios. These include use of specific selective systemic immunosuppressant instead of nonselective immunosuppressant drugs (98), discontinuation of the immunotherapeutic drug followed by a rechallenge with the same drug (109) or rechallenge with PD-L1 inhibitors after intolerable irAEs with anti PD-1 agents (110). In a comprehensive retrospective study of patients treated with ICI, $28.8 \%$ of $24,079 \mathrm{irAE}$ cases represented recurrence of the same irAE after discontinuation and rechallenge with the same ICI. Colitis, hepatitis, and pneumonitis were associated with a higher recurrence rate, while adrenal events were associated with a lower recurrence rate (109). However, none of these strategies have been rigorously tested in patients with SOT and $\mathrm{AD}$.

\section{Enhanced toxicities when combined with other agents}

Combination of immunotherapy with other agents may enhance efficacy but toxicity is also increased (111). Immunotherapy can be combined effectively with other immunotherapeutic drugs, chemotherapy, targeted therapy, and radiotherapy (25). The use of immunotherapy as a single agent may be constrained by the several factors existing in the tumor microenvironment, such as insufficient $\mathrm{T}$ cells from the naive repertoire, inadequate available neoantigen, or immunosuppressive networks which protects the immunogenic tissue from immune attacks. Combining cancer therapies could strengthen the immune response by inducing immunogenicity, alleviating tumor-induced immunosuppression associated with chemotherapy, or rendering tumors more susceptible to cytotoxic $\mathrm{T}$ cell attack (111). In patients with melanoma, five-year OS with combination of ipilimumab with nivolumab was $52 \%$ as compared to $44 \%$ with single agent nivolumab. However, combination treatment also resulted in a higher grade $3 / 4$ treatment related adverse events (59\% vs. 23\%) (112). It is also unknown whether treatment with a PD-1 inhibitor first, followed by treating patients at progression with nivolumab/ipilimumab, can achieve the same OS with less toxicities.

While several combinations of BRAF and MEK inhibitors (BRAF/MEKi) and immunotherapy (mainly anti CTLA-4 and anti PD-1 therapies) increased OS in melanoma patients (113), phase I trials of BRAF/ MEKi with ipilimumab were halted due to unacceptable toxicities. A trial investigating the combination of BRAF inhibitor, vemurafenib plus ipilimumab was halted due to hepatotoxicity (114). The combination of dabrafenib, trametinib, and ipilimumab led to colon perforation in two of the seven patients treated in the phase I trial, while excluding trametinib resulted in no such toxicity (115). In a case report of a melanoma patient treated with sequential BRAF/MEKi (dabrafenib plus trametinib) followed by the ipilimumab, the patient had a complete resolution of the tumor, but died due to fatal gastrointestinal toxicity (113). Contemporary data sets of triplet therapy now show better safety of PD-1 inhibitor combined with BRAF/MEKi (116).

The efficacy and toxicity of immunotherapy combined with other therapies depends on the drug, and relative timing of the combination (117). The critical challenges associated with combining cancer therapy are incorporating immunotherapy into adjuvant and neoadjuvant treatments, determining the accurate dose, duration of treatment, selecting appropriate biomarkers, and designing new surrogate endpoints that accurately define OS benefit at treatment initiation (25). Finally, early identification and treatment of myelosuppression and irAEs associated with disease management with chemo-immunotherapy are necessary, as early treatment enhances survival (111).

\section{HPD}

Despite the success of cancer immunotherapy in demonstrating efficacy across multiple cancer types, it has also been implicated in accelerating disease progression, a concept known as HPD. Notably, this is not as common with other cancer therapeutics $(118,119)$. In a multicenter, retrospective study comparing the incidence of HPD in lung cancer patients receiving ICI therapy versus chemotherapy, HPD occurred in $14 \%$ of patients treated with immunotherapy $(\mathrm{N}=406)$, compared to $5 \%$ of patients receiving chemotherapy $(\mathrm{N}=59)(120)$. The rate of ICIassociated HPD was as high as $10-30 \%$ in retrospective studies (120-122). Variation in the incidence of HPD may result from lack of a unified definition for HPD including or not accounting for non-target lesions like malignant effusions, bone metastases, or new disease sites. Many of the studies were tumor type-specific, and lack of baseline imaging excluded many patients from analysis $(118,123)$. In 
addition, some patients did not meet radiological criteria for HPD because they experienced rapid clinical deterioration with immunotherapy and could not be evaluated by CT $\operatorname{scan}(120,121)$.

Understanding and assessing HPD is further complicated by a phenomenon called pseudoprogression, which refers to transient radiographic worsening (enlarging and/or new tumor lesions) prior to a regression or successful treatment response. This is thought to stem from an early influx of tumor-infiltrating lymphocytes propagating an early but temporary increase in tumor dimensions (124-126). The crucial challenge for oncologists is to distinguish pseudoprogression (affecting less than 10\% of patients (126-128) from standard progression and HPD as this frequently leads to unnecessary discontinuation of a beneficial treatment.

Clinical studies aimed at making this distinction have found HPD to be associated with increased age (121), mutations in a variety of oncogenes (129), higher lactate dehydrogenase (LDH) concentration in the serum (122), female sex (130), prior irradiation of the tumor area (131), presence of liver metastasis or more than two metastatic sites (132), MDM2/MDM4 and EGFR genetic alterations $(133,134)$. However, most study results were not replicated by other studies (135). There is an urgent need for a consensus on a unified definition of HPD so that studies across tumor types can be compared or pooled to achieve a higher statistical power (118).

\section{Lethal or worse outcomes}

Although generally rare with PD-1 inhibitors, some immunotherapeutics resulted in worse and even fatal outcomes in some clinical trials for hematologic malignancies. One example is the chimeric antigen receptor (CAR) T-cell therapy, which is Food and Drug Association (FDA)approved for refractory or relapse (R/R) B cell precursor acute lymphoblastic leukemia (ALL) in pediatric patients and young adults and for R/R large B cell lymphoma in adults. Despite a $92 \%$ response rate in ALL $(136,137)$, in 2016, 5 adult ALL patients died while receiving JCAR015 CAR T-cell therapy. In 2017, a death also resulted in a patient who received UCART123 CAR T-cell therapy during a phase I trial for leukemia. These deaths were associated with the development of neurotoxicity/cerebral edema and cytokine release syndrome (CRS) (138). CRS is an extreme systemic inflammatory response that can progress to sepsis and multiple organ failure (139).
Furthermore, in 2017, the FDA halted two KEYNOTE phase III trials involving pembrolizumab in patients with multiple myeloma due to disproportionately higher deaths in the treatment group compared to controls (138).

The KEYNOTE-183 phase III trial studied pembrolizumab in combination with pomalidomide and low-dose dexamethasone in 249 patients with relapsed/ refractory multiple myeloma. Twenty-nine (23\%) deaths (16 from progressive disease and 13 from adverse events) occurred in the triple therapy group, compared with 21 (17\%) deaths (18 from progressive disease and 3 from adverse events) in the pomalidomide with dexamethasone control group $(138,140)$. The KEYNOTE-185 phase III studied pembrolizumab in combination with lenalidomide and low-dose dexamethasone for newly diagnosed treatment naive multiple myeloma. Nineteen (13\%) (6 because of disease progression and 13 because of adverse events) deaths occurred with triple therapy, compared with $9(6 \%)$ deaths (one because of disease progression and 8 because of adverse events) in the group receiving only lenalidomide and dexamethasone $(138,141)$.

Treatment-related deaths during these trials were attributed to serious adverse effects such as cardiac arrest and failure, large intestine perforation, sepsis, multiple organ failure, Stevens-Johnson syndrome, pulmonary embolism, and respiratory tract infections $(138,140)$. It is not clear if the deaths resulted from the interaction of PD-1 inhibitor with pomalidomide or lenalidomide, or with other concurrent medications.

\section{Variation in response pattern among patients}

Nivolumab has demonstrated activity and favorable safety profile in pediatric patients with glioblastoma in a Phase III clinical trial (CheckMate-143) (142). All adverse events in the 10 nivolumab-treated patients were grade 1 or 2 , with fatigue and nausea as the most common adverse events (143). Despite these safety data, a pediatric patient with glioblastoma treated with nivolumab died from progressive cerebral edema after third treatment (142). By contrast, two pediatric patients with glioblastoma treated with nivolumab experienced transient cerebral edema that subsided with repeated doses and addition of dexamethasone $(142,144)$. Another example of variable patient response includes nivolumab for adult T-cell leukemia-lymphoma (ATLL) in which some patients experienced rapid progression while most did not $(145,146)$. Different disease variants may have accounted for the disparate responses. 
Analysis of three patients who experienced rapid ATLL expansion after PD-1 blockade demonstrated association with tumor-resident regulatory $\mathrm{T}$ cells (Tregs), independent of the unique subtype of the atypical cell present in each patient (147). More studies are clearly needed to understand the variation in patients' adverse responses to immunotherapy.

\section{Financial toxicity}

The cost of immunotherapy is approximately $\$ 100,000$ per patient annually (148). This amount exceeds the median U.S. household income and recently FDA-approved immunotherapeutic drugs are often triple the cost of drugs approved in previous years (149). For instance, the CAR $\mathrm{T}$ cell therapy Tisagenlecleucel which was FDA-approved for treatment of R/R B-cell ALL in patients $\leq 25$ years old, is associated with improved OS but costs $\$ 475,000$ per treatment course, not including cost of hospital stay and toxicities. Its manufacturer, Novartis, is offering a money back guarantee for patients who do not achieve remission within one month of receiving treatment (150).

Studies suggest that some immunotherapeutic drugs were not more cost-effective than chemotherapy based on the quality-adjusted life years (QALYs) gained for some indicators. Cost-effectiveness analysis (CEA) expresses the potential value of new drugs (compared to previous treatment) per health benefits gained in terms of units of currency. The outcome considers OS and is presented as an incremental cost per QALY (151). In a systematic review of the cost and cost-effectiveness studies of ICI, nivolumab was not cost-effective over chemotherapy for recurrent/ metastatic head/neck cancers (HNCs) and NSCLC. Nivolumab and pembrolizumab were not cost-effective for genitourinary cancers, while ipilimumab monotherapy is less cost-effective than nivolumab, nivolumab/ipilimumab, and pembrolizumab for melanoma (152).

Even though many of the immunotherapeutic drugs were found in analytic studies to be cost-effective, this does not necessarily translate into affordability. This has led to rejection of ICI by British National Institute of Clinical Excellence (NICE) and Australian Pharmaceutical Benefits Advisory Committee (PBAC) for some indications (153). Consequently, it is also important for CEA to be performed before FDA approval of these drugs. We also need a lasting solution to curb the current cancer cost trajectory which is untenable for most healthcare systems in the long run, and to save the numerous patients who are going into bankruptcy due to the financial burden of their cancer care (149).

\section{Conclusions}

Despite dramatic therapeutic successes, immunotherapeutic drugs can be associated with numerous and unpredictable toxicities. Furthermore, there is a knowledge gap relating to how these drugs work in different patient populations with various disease conditions. Further clinical studies are needed to maximize the benefits and minimize the risks of immunotherapy. There is also a need to address the rising costs of cancer care to relieve the negative financial burden on patients. Nonetheless, immunotherapy has undoubtedly changed the treatment landscape of oncology care giving patients a greater potential to attain long term survival, improved quality of life, and less toxic treatment options. However, prospective evidence-based studies are necessary to more accurately understand the variation in patient responses and treatment related adverse events, to obtain the full benefit of these promising new drugs.

\section{Acknowledgments}

Funding: None.

\section{Footnote}

Provenance and Peer Review: This article was commissioned by the editorial office, Annals of Translational Medicine for the series "Cancer Immunotherapy: Recent Advances and Challenges". The article has undergone external peer review.

Conflicts of Interest: All authors have completed the ICMJE uniform disclosure form (available at http:// dx.doi.org/10.21037/atm-20-4750). The series "Cancer Immunotherapy: Recent Advances and Challenges" was commissioned by the editorial office without any funding or sponsorship. US served as the unpaid Guest Editor of the series and serves as an unpaid editorial board member of Annals of Translational Medicine from May 2019 to Apr 2021. Dr. SHL reports consulting for Amgen, Merck, Genmab, Xencor, BMS, research support from BMS, Merck, Vaccinex and contracted research from Neon Therapeutics, Astellas, F Star, Xencor, Merck, Vedanta, Boehringer Ingelheim. The authors have no other conflicts of interest to declare.

Etbical Statement: The authors are accountable for all 
aspects of the work in ensuring that questions related to the accuracy or integrity of any part of the work are appropriately investigated and resolved.

Open Access Statement: This is an Open Access article distributed in accordance with the Creative Commons Attribution-NonCommercial-NoDerivs 4.0 International License (CC BY-NC-ND 4.0), which permits the noncommercial replication and distribution of the article with the strict proviso that no changes or edits are made and the original work is properly cited (including links to both the formal publication through the relevant DOI and the license). See: https://creativecommons.org/licenses/by-nc-nd/4.0/.

\section{References}

1. Postow MA, Sidlow R, Hellmann MD. Immunerelated adverse events associated with immune checkpoint blockade. Longo DL, editor. N Engl J Med 2018;378:158-68.

2. Ardolino L, Joshua A. Immune checkpoint inhibitors in malignancy. Aust Prescr 2019;42:62-7.

3. FDA Approval Timeline of Active Immunotherapies Cancer Research Institute (CRI) [Internet]. [cited 2020 Jun 15]. Available online: https://www.cancerresearch. org/scientists/immuno-oncology-landscape/fda-approvaltimeline-of-active-immunotherapies

4. Zhao L, Cao YJ. Engineered T Cell Therapy for Cancer in the Clinic. Front Immunol 2019;10:2250.

5. Kimiz-Gebologlu I, Gulce-Iz S, Biray-Avci C. Monoclonal antibodies in cancer immunotherapy. Mol Biol Rep 2018;45:2935-40.

6. Kroschinsky F, Stölzel F, von Bonin S, et al. New drugs, new toxicities: Severe side effects of modern targeted and immunotherapy of cancer and their management. Critical Care 2017;21:89.

7. Russell L, Peng KW. The emerging role of oncolytic virus therapy against cancer. Chin Clin Oncol 2018;7:16.

8. Carballido E, Fishman M. Sipuleucel-T: Prototype for Development of Anti-tumor Vaccines. Curr Oncol Rep 2011;13:112-9.

9. Caspi RR. Immunotherapy of autoimmunity and cancer: The penalty for success. Nat Rev Immunol 2008;8:970-6.

10. Waldmann TA. Cytokines in cancer immunotherapy. Cold Spring Harb Perspect Biol 2018;10:a028472.

11. Ji N, Mukherjee N, Morales EE, et al. Percutaneous BCG enhances innate effector antitumor cytotoxicity during treatment of bladder cancer: a translational clinical trial.
Oncoimmunology 2019;8:1614857.

12. Badrinath N, Yoo SY. Recent advances in cancer stem celltargeted immunotherapy. Cancers (Basel) 2019;11:310.

13. Xin Yu J, Hodge JP, Oliva C, et al. Trends in clinical development for PD-1/PD-L1 inhibitors. Nat Rev Drug Discov 2020;19:163-4.

14. Naidoo J, Page DB, Li BT, et al. Toxicities of the antiPD-1 and anti-PD-L1 immune checkpoint antibodies. Ann Oncol 2015;26:2375-91.

15. Abdel-Wahab N, Shah M, Suarez-Almazor ME. Adverse events associated with immune checkpoint blockade in patients with cancer: A systematic review of case reports. PLoS One 2016;11:e0160221.

16. Bertrand A, Kostine M, Barnetche T, et al. Immune related adverse events associated with anti-CTLA-4 antibodies: Systematic review and meta-analysis. BMC Med 2015;13:211.

17. Boutros C, Tarhini A, Routier E, et al. Safety profiles of anti-CTLA-4 and anti-PD-1 antibodies alone and in combination. Nat Rev Clin Oncol 2016;13:473-86.

18. Johnson DB, Friedman DL, Berry E, et al. Survivorship in immune therapy: Assessing chronic immune toxicities, health outcomes, and functional status among long-term ipilimumab survivors at a single referral center. Cancer Immunol Res 2015;3:464-9.

19. Larkin J, Chiarion-Sileni V, Gonzalez R, et al. Combined nivolumab and ipilimumab or monotherapy in untreated Melanoma. N Engl J Med 2015;373:23-34.

20. Abdel-Wahab N, Safa H, Abudayyeh A, et al. Checkpoint inhibitor therapy for cancer in solid organ transplantation recipients: An institutional experience and a systematic review of the literature. J Immunother Cancer 2019;7:106.

21. Dulos J, Carven GJ, Van Boxtel SJ, et al. PD-1 blockade augments Th1 and Th17 and suppresses Th2 responses in peripheral blood from patients with prostate and advanced melanoma cancer. J Immunother 2012;35:169-78.

22. von Euw E, Chodon T, Attar N, et al. CTLA4 blockade increases Th17 cells in patients with metastatic melanoma. J Transl Med 2009;7:35.

23. Cadranel J, Canellas A, Matton L, et al. Pulmonary complications of immune checkpoint inhibitors in patients with nonsmall cell lung cancer. Eur Respir Rev 2019;28:190058.

24. Michot JM, Bigenwald C, Champiat S, et al. Immunerelated adverse events with immune checkpoint blockade: A comprehensive review. Eur J Cancer 2016;54:139-48.

25. Emens LA, Ascierto PA, Darcy PK, et al. Cancer immunotherapy: Opportunities and challenges in 
the rapidly evolving clinical landscape. Eur J Cancer 2017;81:116-29.

26. Pillai RN, Behera M, Owonikoko TK, et al. Comparison of the toxicity profile of PD-1 versus PD-L1 inhibitors in non-small cell lung cancer: A systematic analysis of the literature. Cancer 2018;124:271-7.

27. Xu C, Chen YP, Du XJ, et al. Comparative safety of immune checkpoint inhibitors in cancer: Systematic review and network meta-analysis. BMJ 2018;363:k4226.

28. Wang DY, Salem JE, Cohen JV, et al. Fatal Toxic Effects Associated With Immune Checkpoint Inhibitors: A Systematic Review and Meta-analysis. JAMA Oncol 2018;4:1721-8.

29. Herbst RS, Baas P, Kim DW, et al. Pembrolizumab versus docetaxel for previously treated, PD-L1-positive, advanced non-small-cell lung cancer (KEYNOTE-010): A randomised controlled trial. Lancet 2016;387:1540-50.

30. Paz-Ares L, Luft A, Vicente D, et al. Pembrolizumab plus chemotherapy for squamous non-small-cell lung cancer. $\mathrm{N}$ Engl J Med 2018;379:2040-51.

31. Gandhi L, Rodríguez-Abreu D, Gadgeel S, et al. Pembrolizumab plus chemotherapy in metastatic nonsmall-cell lung cancer. N Engl J Med 2018;378:2078-92.

32. Shitara K, Özgüroğlu M, Bang YJ, et al. Pembrolizumab versus paclitaxel for previously treated, advanced gastric or gastro-oesophageal junction cancer (KEYNOTE-061): a randomised, open-label, controlled, phase 3 trial. Lancet 2018;392:123-33.

33. Ferris RL, Blumenschein G, Fayette J, et al. Nivolumab for recurrent squamous-cell carcinoma of the head and neck. N Engl J Med 2016;375:1856-67.

34. Motzer RJ, Tannir NM, McDermott DF, et al. Nivolumab plus Ipilimumab versus Sunitinib in advanced renal-cell carcinoma. N Engl J Med 2018;378:1277-90.

35. Powles T, Durán I, van der Heijden MS, et al. Atezolizumab versus chemotherapy in patients with platinum-treated locally advanced or metastatic urothelial carcinoma (IMvigor211): a multicentre, open-label, phase 3 randomised controlled trial. Lancet 2018;391:748-57.

36. Bellmunt J, De Wit R, Vaughn DJ, et al. Pembrolizumab as second-line therapy for advanced urothelial carcinoma. N Engl J Med 2017;376:1015-26.

37. Borghaei H, Paz-Ares L, Horn L, et al. Nivolumab versus docetaxel in advanced nonsquamous non-small-cell lung cancer. N Engl J Med 2015;373:1627-39.

38. De Velasco G, Je Y, Bossé D, et al. Comprehensive metaanalysis of key immune-related adverse events from CTLA-4 and PD-1/PD-L1 inhibitors in cancer patients.
Cancer Immunol Res 2017;5:312-8.

39. Wang Y, Zhou S, Yang F, et al. Treatment-Related Adverse Events of PD-1 and PD-L1 Inhibitors in Clinical Trials: A Systematic Review and Meta-analysis. JAMA Oncol 2019;5:1008-19.

40. Tabata MM, Choi S, Hirotsu K, et al. Cardiotoxicity associated with immune checkpoint inhibitors in cutaneous oncology. J Am Acad Dermatol 2020;82:986-94.

41. Hughes J, Vudattu N, Sznol M, et al. Precipitation of autoimmune diabetes with anti-PD-1 immunotherapy. Diabetes Care 2015;38:e55-7.

42. Smati S, Buffier P, Bouillet B, et al. Expert opinion on immunotherapy induced diabetes. Ann Endocrinol (Paris) 2018;79:545-9.

43. Joshi MN, Whitelaw BC, Palomar MTP, et al. Immune checkpoint inhibitor-related hypophysitis and endocrine dysfunction: clinical review Clin Endocrinol (Oxf) 2016;85:331-9.

44. Byun DJ, Wolchok JD, Rosenberg LM, et al. Cancer immunotherapy-immune checkpoint blockade and associated endocrinopathies. Nat Rev Endocrinol 2017;13:195-207.

45. Haissaguerre M, Hescot S, Bertherat J, et al. Expert opinions on adrenal complications in immunotherapy. Ann Endocrinol (Paris) 2018;79:539-44.

46. de Filette JM, Pen JJ, Decoster L, et al. Immune checkpoint inhibitors and type 1 diabetes mellitus: A case report and systematic review. Eur J Endocrinol 2019;181:363-74.

47. Cheema A, Makadia B, Karwadia T, et al. Autoimmune Diabetes Associated With Pembrolizumab: A Review of Published Case Reports. World J Oncol 2018;9:1-4.

48. Scott ES, Long GV, Guminski A, et al. The spectrum, incidence, kinetics and management of endocrinopathies with immune checkpoint inhibitors for metastatic melanoma. Eur J Endocrinol 2018;178:173-80.

49. Sznol M, Postow MA, Davies MJ, et al. Endocrinerelated adverse events associated with immune checkpoint blockade and expert insights on their management. Cancer Treat Rev 2017;58:70-6.

50. Stamatouli AM, Quandt Z, Perdigoto AL, et al. Collateral damage: Insulin-dependent diabetes induced with checkpoint inhibitors. Diabetes 2018;67:1471-80.

51. Ferrari SM, Fallahi P, Galetta F, et al. Thyroid disorders induced by checkpoint inhibitors. Rev Endocr Metab Disord 2018;19:325-33.

52. Wang A, Xu Y, Fei Y, et al. The role of immunosuppressive agents in the management of severe and refractory 
immune-related adverse events. Asia Pac J Clin Oncol 2020;16:201-10.

53. Valpione S, Pasquali S, Campana LG, et al. Sex and interleukin-6 are prognostic factors for autoimmune toxicity following treatment with anti-CTLA4 blockade. J Transl Med 2018;16:94.

54. Chaput N, Lepage P, Coutzac C, et al. Baseline gut microbiota predicts clinical response and colitis in metastatic melanoma patients treated with ipilimumab. Ann Oncol 2017;28:1368-79.

55. Tarhini AA, Zahoor H, Lin Y, et al. Baseline circulating IL-17 predicts toxicity while TGF- $\beta 1$ and IL- 10 are prognostic of relapse in ipilimumab neoadjuvant therapy of melanoma. J Immunother Cancer 2015;3:39.

56. Tanaka R, Okiyama N, Okune M, et al. Serum level of interleukin-6 is increased in nivolumab-associated psoriasiform dermatitis and tumor necrosis factor- $\alpha$ is a biomarker of nivolumab recativity. J Dermatol Sci 2017;86:71-3.

57. Fujimura T, Sato Y, Tanita K, et al. Serum levels of soluble CD163 and CXCL5 may be predictive markers for immune-related adverse events in patients with advanced melanoma treated with nivolumab: A pilot study. Oncotarget 2018;9:15542-51.

58. Kimbara S, Fujiwara Y, Iwama S, et al. Association of antithyroglobulin antibodies with the development of thyroid dysfunction induced by nivolumab. Cancer Sci 2018;109:3583-90.

59. Gowen MF, Giles KM, Simpson D, et al. Baseline antibody profiles predict toxicity in melanoma patients treated with immune checkpoint inhibitors. J Transl Med 2018;16:82.

60. Dubin K, Callahan MK, Ren B, et al. Intestinal microbiome analyses identify melanoma patients at risk for checkpoint-blockade-induced colitis. Nat Commun 2016;7:10391.

61. Downey SG, Klapper JA, Smith FO, et al. Prognostic factors related to clinical response in patients with metastatic melanoma treated by CTL-associated antigen-4 blockade. Clin Cancer Res 2007;13:6681-8.

62. Freeman-Keller M, Kim Y, Cronin H, et al. Nivolumab in resected and unresectable metastatic melanoma: Characteristics of immune-related adverse events and association with outcomes. Clin Cancer Res 2016;22:886-94.

63. Teulings HE, Limpens J, Jansen SN, et al. Vitiligo-like depigmentation in patients with stage III-IV melanoma receiving immunotherapy and its association with survival: A systematic review and meta-analysis. J Clin Oncol
2015;33:773-81.

64. Sanlorenzo M, Vujic I, Daud A, et al. Pembrolizumab cutaneous adverse events and their association with disease progression. JAMA Dermatol 2015;151:1206-12 .

65. Horvat TZ, Adel NG, Dang TO, et al. Immune-related adverse events, need for systemic immunosuppression, and effects on survival and time to treatment failure in patients with melanoma treated with ipilimumab at memorial sloan kettering cancer center. J Clin Oncol 2015;33:3193-8.

66. Weber JS, Hodi FS, Wolchok JD, et al. Safety profile of nivolumab monotherapy: A pooled analysis of patients with advanced melanoma. J Clin Oncol 2017;35:785-92.

67. Kyi C, Hellmann MD, Wolchok JD, et al. Opportunistic infections in patients treated with immunotherapy for cancer. J Immunother Cancer 2014;2:19.

68. Fishman JA, Hogan JI, Maus MV. Inflammatory and infectious syndromes associated with cancer immunotherapies. Clin Infect Dis 2019;69:909-20.

69. Anastasopoulou A, Ziogas DC, Samarkos M, et al. Reactivation of tuberculosis in cancer patients following administration of immune checkpoint inhibitors: Current evidence and clinical practice recommendations. J Immunother Cancer 2019;7:239.

70. Barber DL, Sakai S, Kudchadkar RR, et al. Tuberculosis following PD-1 blockade for cancer immunotherapy. Sci Transl Med 2019;11:eaat2702.

71. Chu YC, Fang KC, Chen HC, et al. Pericardial Tamponade Caused by a Hypersensitivity Response to Tuberculosis Reactivation after Anti-PD-1 Treatment in a Patient with Advanced Pulmonary Adenocarcinoma. J Thorac Oncol 2017;12:e111-e114.

72. He W, Zhang X, Li W, et al. Activated pulmonary tuberculosis in a patient with melanoma during PD-1 inhibition: A case report. Onco Targets Ther 2018;11:7423-7.

73. Jensen KH, Persson G, Bondgaard AL, et al. Development of pulmonary tuberculosis following treatment with antiPD-1 for non-small cell lung cancer. Acta Oncologica 2018;57:1127-8.

74. Picchi H, Mateus C, Chouaid C, et al. Infectious complications associated with the use of immune checkpoint inhibitors in oncology: reactivation of tuberculosis after anti PD-1 treatment. Clin Microbiol Infect 2018;24:216-8.

75. Elkington PT, Bateman AC, Thomas GJ, et al. Implications of tuberculosis reactivation after immune checkpoint inhibition. Am J Respir Crit Care Med 2018;198:1451-3. 
76. Tsai CC, Chen JH, Wang YC, et al. Re-activation of pulmonary tuberculosis during anti-programmed death-1 (PD-1) treatment. QJM 2019;112:41-2.

77. Takata S, Koh G, Han Y, et al. Paradoxical response in a patient with non-small cell lung cancer who received nivolumab followed by anti-Mycobacterium tuberculosis agents. J Infect Chemother 2019;25:54-8.

78. Shen L, Gao Y, Liu Y, et al. PD-1/PD-L pathway inhibits M.tb-specific CD4+ T-cell functions and phagocytosis of macrophages in active tuberculosis. Sci Rep 2016;6:38362.

79. Tezera LB, Bielecka MK, Ogongo P, et al. Anti-PD-1 immunotherapy leads to tuberculosis reactivation via dysregulation of TNF- $\alpha$. Elife 2020;9:e52668

80. Berthod G, Lazor R, Letovanec I, et al. Pulmonary sarcoid-like granulomatosis induced by ipilimumab. J Clin Oncol 2012:30:e156-9.

81. Vogel WV, Guislain A, Kvistborg P, et al. Ipilimumabinduced sarcoidosis in a patient with metastatic melanoma undergoing complete remission. J Clin Oncol 2012;30:e7-e10.

82. Montaudié H, Pradelli J, Passeron T, et al. Pulmonary sarcoid-like granulomatosis induced by nivolumab. Br J Dermatol 2017;176:1060-3.

83. Al-Dliw M, Megri M, Shahoub I, et al. Pembrolizumab reactivates pulmonary granulomatosis. Respir Med Case Rep2017;22:126-9.

84. Lainez S, Tissot C, Cottier M, et al. EBUS-TBNA Can Distinguish Sarcoid-Like Side Effect of Nivolumab Treatment from Tumor Progression in Non-Small Cell Lung Cancer. Respiration 2017;94:518-21.

85. Noguchi S, Kawachi H, Yoshida H, et al. Sarcoid-Like Granulomatosis Induced by Nivolumab Treatment in a Lung Cancer Patient. Case Rep Oncol 2018;11:562-6.

86. Cousin S, Italiano A. Molecular pathways: Immune checkpoint antibodies and their toxicities. Clin Cancer Res 2016;22:4550-5.

87. Mitchell MA, Hogan K, Amjadi K. Atezolizumabinduced sarcoid-like granulomatous reaction in a patient with urothelial cell carcinoma. Immunotherapy 2018;10:1189-92.

88. Reule RB, North JP. Cutaneous and pulmonary sarcoidosis-like reaction associated with ipilimumab. J Am Acad Dermatol 2013;69:e272-e273.

89. Murphy KP, Kennedy MP, Barry JE, et al. New-onset mediastinal and central nervous system sarcoidosis in a patient with metastatic melanoma undergoing CTLA4 monoclonal antibody treatment. Oncol Res Treat 2014;37:351-3.
90. Eckert A, Schoeffler A, Dalle S, et al. Anti-CTLA4 monoclonal antibody induced sarcoidosis in a metastatic melanoma patient. Dermatology 2009;218:69-70.

91. Tissot C, Carsin A, Freymond N, et al. Sarcoidosis complicating anti-cytotoxic T-lymphocyte-associated antigen-4 monoclonal antibody biotherapy. Eur Respir J 2013;41:246-7.

92. Koelzer VH, Rothschild SI, Zihler D, et al. Systemic inflammation in a melanoma patient treated with immune checkpoint inhibitors-an autopsy study. J Immunother Cancer 2016;4:13.

93. Reuss JE, Kunk PR, Stowman AM, et al. Sarcoidosis in the setting of combination ipilimumab and nivolumab immunotherapy: A case report \& review of the literature. J Immunother Cancer 2016;4:94.

94. Faviez G, Bousquet E, Rabeau A, et al. Sarcoid-like granulomatosis in cancer patients treated with immune checkpoints inhibitors. Rev Mal Respir 2018;35:963-7.

95. Danlos FX, Pagès C, Baroudjian B, et al. Nivolumabinduced sarcoid-like granulomatous reaction in a patient with advanced melanoma. Chest 2016;149:e133-6.

96. Eggermont AMM, Blank CU, Mandala M, et al. Adjuvant pembrolizumab versus placebo in resected stage III melanoma. N Engl J Med 2018;378:1789-801.

97. Bonifazi M, Bravi F, Gasparini S, et al. Sarcoidosis and cancer risk: Systematic review and meta-analysis of observational studies systematic review and meta-analysis of observational studies. Chest 2015;147:778-91.

98. Haanen J, Ernstoff M, Wang Y, et al. Autoimmune diseases and immune-checkpoint inhibitors for cancer therapy: Review of the literature and personalized riskbased prevention strategy. Ann Oncol 2020;31:724-44.

99. Ros J, Matos I, Martin-Liberal J. Immunotherapy in organ-transplanted cancer patients: efficacy and risk of organ rejection. Ann Oncol 2019;30:1173-7.

100. Fisher J, Zeitouni N, Fan W, et al. Immune checkpoint inhibitor therapy in solid organ transplant recipients: A patient-centered systematic review. J Am Acad Dermatol 2020;82:1490-500.

101.Abdel-Wahab N, Shah M, Lopez-Olivo MA, et al. Use of immune checkpoint inhibitors in the treatment of patients with cancer and preexisting autoimmune disease: A systematic review. Ann Int Med 2018;168:121-30.

102. Frohne CC, Llano EM, Perkovic A, et al. Complete response of metastatic melanoma in a patient with Crohn's disease simultaneously receiving anti-alpha4beta7 and antiPD1 antibodies. J Immunother Cancer 2019; 7:1.

103. Uemura M, Trinh VA, Haymaker C, et al. Selective 
inhibition of autoimmune exacerbation while preserving the anti-tumor clinical benefit using IL-6 blockade in a patient with advanced melanoma and Crohn's disease: A case report. J Hematol Oncol 2016;9:81.

104. Arbour KC, Mezquita L, Long N, et al. Impact of baseline steroids on efficacy of programmed cell death-1 and programmed death-ligand 1 blockade in patients with nonsmall-cell lung cancer. J Clin Oncol 2018;36:2872-8.

105.Hughes MS, Zheng H, Zubiri L, et al. Colitis after checkpoint blockade: A retrospective cohort study of melanoma patients requiring admission for symptom control. Cancer Med 2019;8:4986-99.

106. Ricciuti B, Dahlberg SE, Adeni A, et al. Immune checkpoint inhibitor outcomes for patients with nonsmall-cell lung cancer receiving baseline corticosteroids for palliative versus nonpalliative indications. J Clin Oncol 2019;37:1927-34.

107.Alliance Foundation Trials L. Nivolumab in Patients with Advanced Non-Small Cell Lung Cancer and Preexisting Autoimmune Disease. Clin Identifier 2018; NCT03656627.

108.(NCI) NCI. Nivolumab in Treating Patients with Autoimmune Disorders or Advanced, Metastatic, or Unresectable Cancer. Clin Identifier 2019; NCT03816345.

109. Dolladille C, Ederhy S, Sassier M, et al. Immune Checkpoint Inhibitor Rechallenge after Immune-Related Adverse Events in Patients with Cancer. JAMA Oncol 2020;6:865-71.

110. Swami U, Lenert P, Furqan M, et al. Atezolizumab after Nivolumab-Induced Inflammatory Polyarthritis: Can Anti-PD-L1 Immunotherapy Be Administered after Anti-PD-1-Related Immune Toxicities? J Thorac Oncol 2018:13:e102-e103.

111.Luo Q, Zhang L, Luo C, et al. Emerging strategies in cancer therapy combining chemotherapy with immunotherapy. Cancer Letters 2019;454:191-203.

112.Larkin J, Chiarion-Sileni V, Gonzalez R, et al. Five-year survival with combined nivolumab and ipilimumab in advanced melanoma. N Engl J Med 2019;381:1535-46.

113. Gonzalez-Cao M, Boada A, Teixidó C, et al. Fatal gastrointestinal toxicity with ipilimumab after BRAF/ MEK inhibitor combination in a melanoma patient achieving pathological complete response. Oncotarget 2016;7:56619-27.

114. Ribas A, Hodi FS, Callahan M, et al. Hepatotoxicity with combination of vemurafenib and ipilimumab. New Engl J Med 2013;368:1365-6.

115.Minor DR, Puzanov I, Callahan MK, et al. Severe gastrointestinal toxicity with administration of trametinib in combination with dabrafenib and ipilimumab. Pigment Cell Melanoma Res 2015;28:611-2.

116. Ribas A, Lawrence D, Atkinson V, et al. Combined BRAF and MEK inhibition with PD-1 blockade immunotherapy in BRAF-mutant melanoma. Nat Med 2019;25:936-40.

117. Emens LA, Middleton G. The interplay of immunotherapy and chemotherapy: Harnessing potential synergies. Cancer Immunol Res 2015;3:436-43

118. Popat V, Gerber DE. Hyperprogressive disease: A distinct effect of immunotherapy? J Thorac Dis 2019;11:S262-S265.

119.Kocikowski M, Dziubek K, Parys M. Hyperprogression under immune checkpoint-based immunotherapycurrent understanding, the role of $\mathrm{pd}-1 / \mathrm{pd}-11$ tumourintrinsic signalling, future directions and a potential large animal model. Cancers 2020;12:804.

120. Ferrara R, Mezquita L, Texier M, et al. Hyperprogressive Disease in Patients with Advanced Non-Small Cell Lung Cancer Treated with PD-1/PD-L1 Inhibitors or with Single-Agent Chemotherapy. JAMA Oncol 2018;4:1543-52.

121. Champiat S, Dercle L, Ammari S, et al. Hyperprogressive disease is a new pattern of progression in cancer patients treated by anti-PD-1/PD-L1. Clin Cancer Res 2017;23:1920-8.

122. Kim JY, Lee KH, Kang J, et al. Hyperprogressive disease during anti-PD-1 (PDCD1) / PD-L1 (CD274) therapy: A systematic review and meta-analysis. Cancers (Basel) 2019;11:1699.

123. Frelaut M, Le Tourneau C, Borcoman E. Hyperprogression under immunotherapy. Int J Mol Sci 2019;20:2674.

124. Seymour L, Bogaerts J, Perrone A, et al. iRECIST: guidelines for response criteria for use in trials testing immunotherapeutics. Lancet Oncol 2017;18:e143-e152.

125. Tazdait M, Mezquita L, Lahmar J, et al. Patterns of responses in metastatic NSCLC during PD-1 or PDL-1 inhibitor therapy: Comparison of RECIST 1.1, irRECIST and iRECIST criteria. Eur J Cancer 2018;88:38-47.

126. Wolchok JD, Hoos A, O'Day S, et al. Guidelines for the evaluation of immune therapy activity in solid tumors: Immune-related response criteria. Clin Cancer Res 2009;15:7412-20.

127. Queirolo P, Spagnolo F. Atypical responses in patients with advanced melanoma, lung cancer, renal-cell carcinoma and other solid tumors treated with anti-PD-1 drugs: A systematic review. Cancer Treat Rev 2017;59:71-8.

128. Hodi FS, Hwu WJ, Kefford R, et al. Evaluation of immune-related response criteria and RECIST v1.1 
in patients with advanced melanoma treated with

Pembrolizumab. J Clin Oncol 2016;34:1510-7.

129.Xiong D, Wang Y, Singavi AK, et al. Immunogenomic Landscape Contributes to Hyperprogressive Disease after Anti-PD-1 Immunotherapy for Cancer. iScience 2018;9:258-77.

130.Kanjanapan Y, Day D, Wang L, et al. Hyperprogressive disease in early-phase immunotherapy trials: Clinical predictors and association with immune-related toxicities. Cancer 2019;125:1341-9.

131. Saâda-Bouzid E, Defaucheux C, Karabajakian A, et al. Hyperprogression during anti-PD-1/PD-L1 therapy in patients with recurrent and/or metastatic head and neck squamous cell carcinoma. Ann Oncol 2017;28:1605-11.

132. Chen Y, Hu J, Bu F. Clinical characteristics of hyperprogressive disease in NSCLC after treatment with immune checkpoint inhibitor: a systematic review and meta-analysis. BMC Cancer 2020;20:707.

133. Kato S, Goodman A, Walavalkar V, et al. Hyperprogressors after immunotherapy: Analysis of genomic alterations associated with accelerated growth rate. Clin Cancer Res 2017;23;4242-50.

134. Singavi AK, Menon S, Kilari D, et al. 1140PDPredictive biomarkers for hyper-progression (HP) in response to immune checkpoint inhibitors (ICI) - analysis of somatic alterations (SAs). Ann Oncol 2017;28:v403-v427.

135. Farè E, Sdao S, Damian S, et al. Hyperprogression during immuno-checkpoint inhibitors (ICIs): A clinically significant problem? Ann Oncol Oncol 2018;29:viii430.

136.Zheng PP, Kros JM, Li J. Approved CAR T cell therapies: ice bucket challenges on glaring safety risks and long-term impacts. Drug Discovery Today 2018.;23:1175-82.

137. Miliotou AN, Papadopoulou LC. CAR T-cell Therapy: A New Era in Cancer Immunotherapy. Curr Pharm Biotechnol 2018;19:5-18.

138. Schmidt C. The struggle to do no harm in clinical trials. Nature 2017;552:S74-S75.

139. Shimabukuro-Vornhagen A, Gödel P, Subklewe M, et al. Cytokine release syndrome. J Immunother Cancer 2018;6:56.

140. Mateos MV, Blacklock H, Schjesvold F, et al. Pembrolizumab plus pomalidomide and dexamethasone for patients with relapsed or refractory multiple myeloma (KEYNOTE-183): a randomised, open-label, phase 3 trial. Lancet Haematol 2019;6:e459-e469.

141. Usmani SZ, Schjesvold F, Oriol A, et al. Pembrolizumab plus lenalidomide and dexamethasone for patients with treatment-naive multiple myeloma (KEYNOTE-185): a randomised, open-label, phase 3 trial. Lancet Haematol 2019;6:e448-e458.

142.Zhu X, McDowell MM, Newman WC, et al. Severe cerebral edema following nivolumab treatment for pediatric glioblastoma: Case report. J Neurosurg Pediatr 2017;19:249-53.

143.Sampson JH, Vlahovic G, Sahebjam S, et al. Preliminary safety and activity of nivolumab and its combination with ipilimumab in recurrent glioblastoma (GBM): CHECKMATE-143. J Clin Oncol 2015;33:3010.

144. Bouffet E, Larouche V, Campbell BB, et al. Immune checkpoint inhibition for hypermutant glioblastoma multiforme resulting from germline biallelic mismatch repair deficiency. J Clin Oncol 2016;34:2206-11.

145. Ratner L, Waldmann TA, Janakiram M, et al. Rapid progression of adult T-cell leukemia-lymphoma after PD-1 inhibitor therapy. New Engl J Med 2018;378:1947-8.

146. Ishitsuka K, Utsunomiya A, Ishida T. PD-1 Inhibitor Therapy in Adult T-Cell Leukemia-Lymphoma. New Engl J Med 2018;379:695-7.

147. Rauch DA, Conlon KC, Janakiram M, et al. Rapid progression of adult T-cell leukemia/lymphoma as tumor-infiltrating Tregs after PD-1 blockade. Blood 2019;134:1406-14.

148. Dranitsaris G, Zhu X, Adunlin G, et al. Cost effectiveness vs. affordability in the age of immuno-oncology cancer drugs. Expert Rev Pharmacoecon Outcomes Res 2018;18:351-7.

149. Cressman S, Browman GP, Hoch JS, et al. A Time-Trend Economic Analysis of Cancer Drug Trials. Oncologist 2015;20:729-36.

150.Jain T, Litzow MR. No free rides: Management of toxicities of novel immunotherapies in ALL, including financial. Hematology Am Soc Hematol Educ Program 2018;2018:25-34.

151. Drummond MF, Stoddart GL TG. Methods for the eco-nomic evaluation of health care programs. Oxford, England: Oxford Univ. Press; 2005.

152. Verma V, Sprave T, Haque W, et al. A systematic review of the cost and cost-effectiveness studies of immune checkpoint inhibitors. J Immunother Cancer 2018;6:128.

153.Kim H, Liew D, Goodall S. Cost-effectiveness and financial risks associated with immune checkpoint inhibitor therapy. Br J Clin Pharmacol 2020;86:1703-10.

Cite this article as: Okwundu N, Grossman D, Hu-Lieskovan S, Grossmann KF, Swami U. The dark side of immunotherapy. Ann Transl Med 2021;9(12):1041. doi: 10.21037/atm-20-4750 